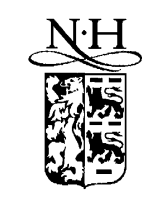

ELSEVIER

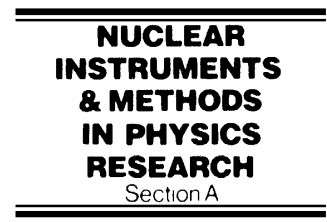

www.elsevier.com/locate/nima

\title{
Development of large area and of position-sensitive timing RPCs
}

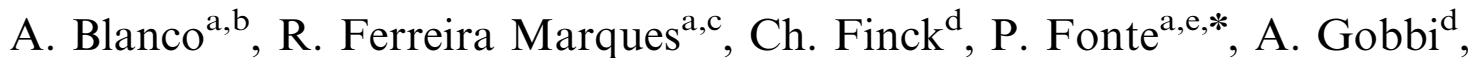

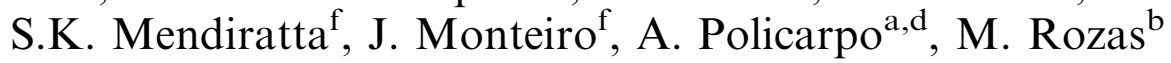 \\ ${ }^{a}$ Dep. De Fisica, Universidade de Coimbra, LIP, P-3004-516 Coimbra, Portugal \\ ${ }^{\mathrm{b}}$ GENP, Dept. Fisica de Particulas, Univ. Santiago de Compostela, Spain \\ ${ }^{\mathrm{c}}$ Departamento de Fisica da Universidade de Coimbra, Portugal \\ ${ }^{\mathrm{d}}$ GSI, Darmstadt, Germany \\ e ISEC, Coimbra, Portugal \\ ${ }^{\mathrm{f}}$ Departamento de Fisica da Universidade de Aveiro, Portugal
}

\begin{abstract}
Resistive Plate Chambers (RPC) made with glass and metal electrodes forming accurately spaced gas gaps of a few hundred micrometers have reached timing accuracies below $50 \mathrm{ps} \sigma$ with efficiencies above $99 \%$ for MIPs. This type of detector, operating at atmospheric pressure with non-flammable gases, seems well suited for large TOF systems, providing performances comparable to the scintillator-based TOF technology with significantly lower price per channel and being compatible with magnetic fields.

In this work, we report recent developments of the timing RPC technology, including a large area counter $\left(0.16 \mathrm{~m}^{2}\right)$ and smaller, position-sensitive, single-gap counters. The latter devices are aimed to be applied in small and accurate TOF systems using a multilayer structure.

The large counter has shown a timing resolution between 50 and $75 \mathrm{ps} \sigma$ over the whole active area, with $96 \%$ efficiency and very small timing tails, along with a longitudinal position-resolution of $1.2 \mathrm{~cm} \sigma$. The single-gap counters had a timing resolution of $55 \mathrm{ps} \sigma$ and a bi-dimensional position-resolution better than $3 \mathrm{~mm}$ FWHM. It was calculated that a 4-layer configuration of such counters would yield a time resolution of $33 \mathrm{ps} \sigma$ essentially free from timing tails. (C) 2002 Elsevier Science B.V. All rights reserved.
\end{abstract}

PACS: $29.40 . \mathrm{Cs}$

Keywords: Gaseous detectors; Time resolution; TOF; RPC

\section{Introduction}

The development of timing Resistive Plate Chambers (RPCs) [1] opened the possibility to

*Corresponding author. Tel.: +351-239-833-465; fax: + 35123-98-29-158.

E-mail address: fonte@lipc.fis.uc.pt (P. Fonte). build fine-granularity high-resolution TOF systems at a quite reduced cost per channel when compared to the standard scintillator-based technology.

Previous work yielded a timing resolution better than 50 ps $\sigma$ at $99 \%$ efficiency for single four-gap chambers [2] and an average timing resolution of 88 ps $\sigma$ at an average efficiency of $97 \%$ for a 32 


\section{Top view}
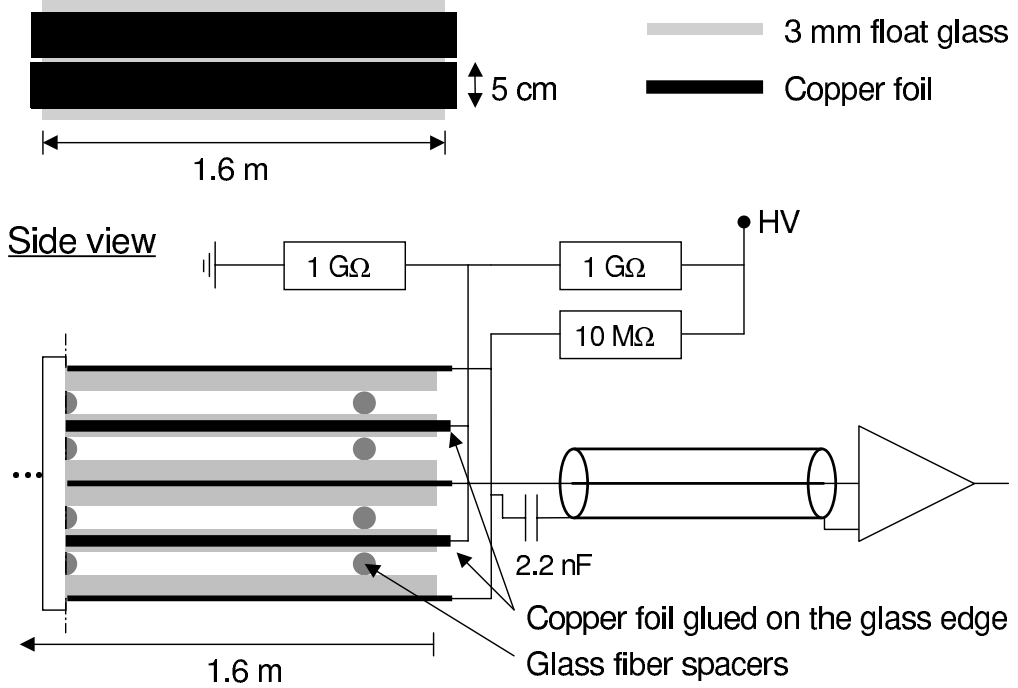

Fig. 1. Schematic diagrams of the large counter construction and wiring.

channel system [3]. It has also been shown that each amplifying gap of $0.3 \mathrm{~mm}$ thickness has a detection efficiency close to $75 \%$ and that the avalanche develops under the influence of a strong space charge effect [4]. A Monte Carlo model of the avalanche development reproduced well the observed data, confirming the dominant role of space charge effects in these detectors [5].

Although timing RPCs have so far been built with relatively small active areas per electronic readout channel (on the order of $10 \mathrm{~cm}^{2}$ ), compatible with the high-multiplicity requirements of High Energy Heavy Ion Physics, there are a number of possible applications in lower multiplicity environments $[6,7]$ for more coarsely segmented counters.

Having in view such applications, in this paper we will briefly describe the structure and performance of two counter types: a large counter, with an active area of $160 \times 10 \mathrm{~cm}^{2}$, readout by only 2 or 4 electronic channels and smaller $\left(16 \mathrm{~cm}^{2}\right)$ single-gap position-sensitive counters, to be used in multilayer configurations for small and accurate TOF systems. The position information would allow a better measurement of the particle's trajectory and if needed could be used to correct offline for any inhomogeneities in the gas gap spacing.

\section{Description of the detectors}

\subsection{Large counter}

The detector was built from $3 \mathrm{~mm}$ thick floatglass plates with an area of $160 \times 12 \mathrm{~cm}^{2}$ and a measured bulk resistivity of $2 \times 10^{12} \Omega \mathrm{cm}$. Four amplifying gaps were accurately defined by $0.3 \mathrm{~mm}$ diameter glass fibres placed between the glass plates, beneath pressing bars.

A schematic diagram of the detector construction and wiring can be seen in Fig. 1 and further details can be found in Ref. [8].

\subsection{Single-gap counters}

The counters were made from commercial tainted glass ${ }^{1}$ that was thinned to $2 \mathrm{~mm}$ thickness and had the inner face lapped to a flatness of $\sim 1 \mu \mathrm{m}$, as estimated by optical interferometry. A $0.5 \mathrm{~mm}$ deep trench was carved around each corner of the glass plate, forming a pillar that supported $0.3 \mathrm{~mm}$ thick high-resistivity glass

\footnotetext{
${ }^{1}$ SCHOTT ATHERMAL ${ }^{\mathrm{TM}}$
} 


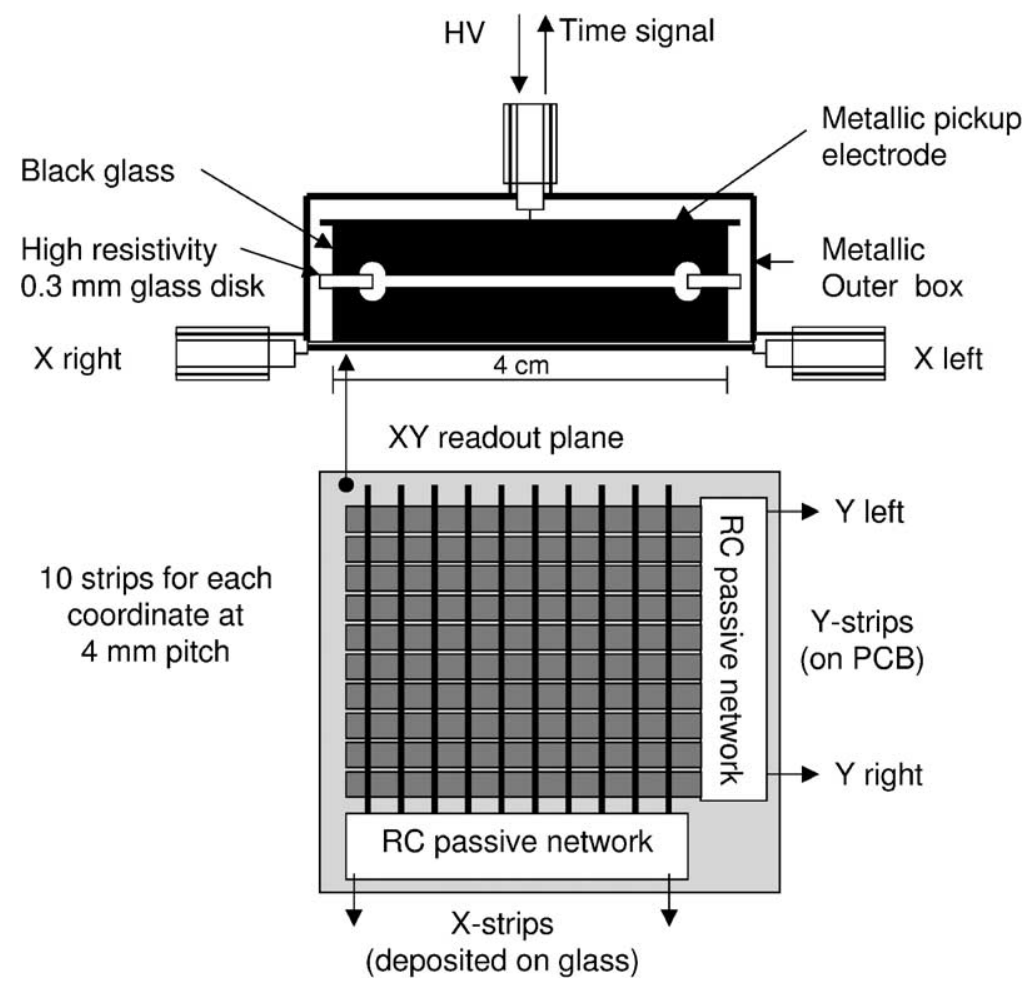

Fig. 2. Schematic diagrams of the single-gap position-sensitive counter construction and wiring.

spacer disks. The trench protected the spacer edge, forming an essentially noiseless spacing structure. The avalanche signals were induced between a metallic electrode on the high-voltage side and a position-sensitive one on the ground side. Further details about the chamber construction and wiring are schematically shown in Fig. 2.

All counters, high-voltage distribution network and preamplifiers were placed inside gas-tight aluminium enclosures kept under a continuous flow of a non-flammable gas mixture consisting of $85 \% \mathrm{C}_{2} \mathrm{H}_{2} \mathrm{~F}_{4}{ }^{2}+10 \% \mathrm{SF}_{6}+5 \%$ iso- $\mathrm{C}_{4} \mathrm{H}_{10}$ [9], at a flow rate close to $100 \mathrm{~cm}^{3} / \mathrm{min}$.

\subsection{Test set-up and data acquisition}

The tests were made at the CERN PS using a secondary beam (T11) of $3.5 \mathrm{GeV} / c$ particles,

\footnotetext{
${ }^{2}$ Commercially known as R134a.
}

mainly negative pions, during August-September 2000.

A pair of plastic scintillation counters provided the reference time information with a resolution close to $35 \mathrm{ps} \sigma$ and defined a coincidence (trigger) area of $2 \times 2 \mathrm{~cm}^{2}$.

The time and charge readout of all devices was accomplished by custom-made frontend electronics, featuring measured timing and charge resolutions of, respectively, $10 \mathrm{ps} \sigma$ and $3.2 \mathrm{fC} \sigma$ [10]. The time information was digitised by a LeCroy 2229 TDC and a LeCroy $2249 \mathrm{w}$ charge-sensitive ADC digitised the corresponding charge information. The position information of the smaller chambers was also readout by custom-made circuitry and digitised by a peak-sensitive LeCroy 2249b ADC.

Further details about the test set-up, data acquisition and analysis can be found elsewhere [2], [8]. 


\section{Results}

\subsection{Large counter}

In Fig. 3a, b and c we show, respectively, the counter efficiency for MIPs, the timing resolution and the amount of timing tails as a function of the position of the centre of the trigger region along each strip.

The efficiency values lay between $95 \%$ and $98 \%$ and the timing resolution ranges from 50 to $75 \mathrm{ps} \sigma$ over the whole active area. Timing tails, defined as the fraction of events that deviate from the average time by more than $300 \mathrm{ps}$, were smaller than $2 \%$ ( $1 \%$ for strip B).

The combination of both strip signals into a single amplifier at each end of the detector, doubling the active area per amplifier, caused absolutely no degradation in either the efficiency or the time resolution of the device. Also, no clear influence from the spacers could be found in a finestep scan (strip A from 0 to $20 \mathrm{~cm}$ ).

The avalanche position along the strips could be estimated from the time difference between the
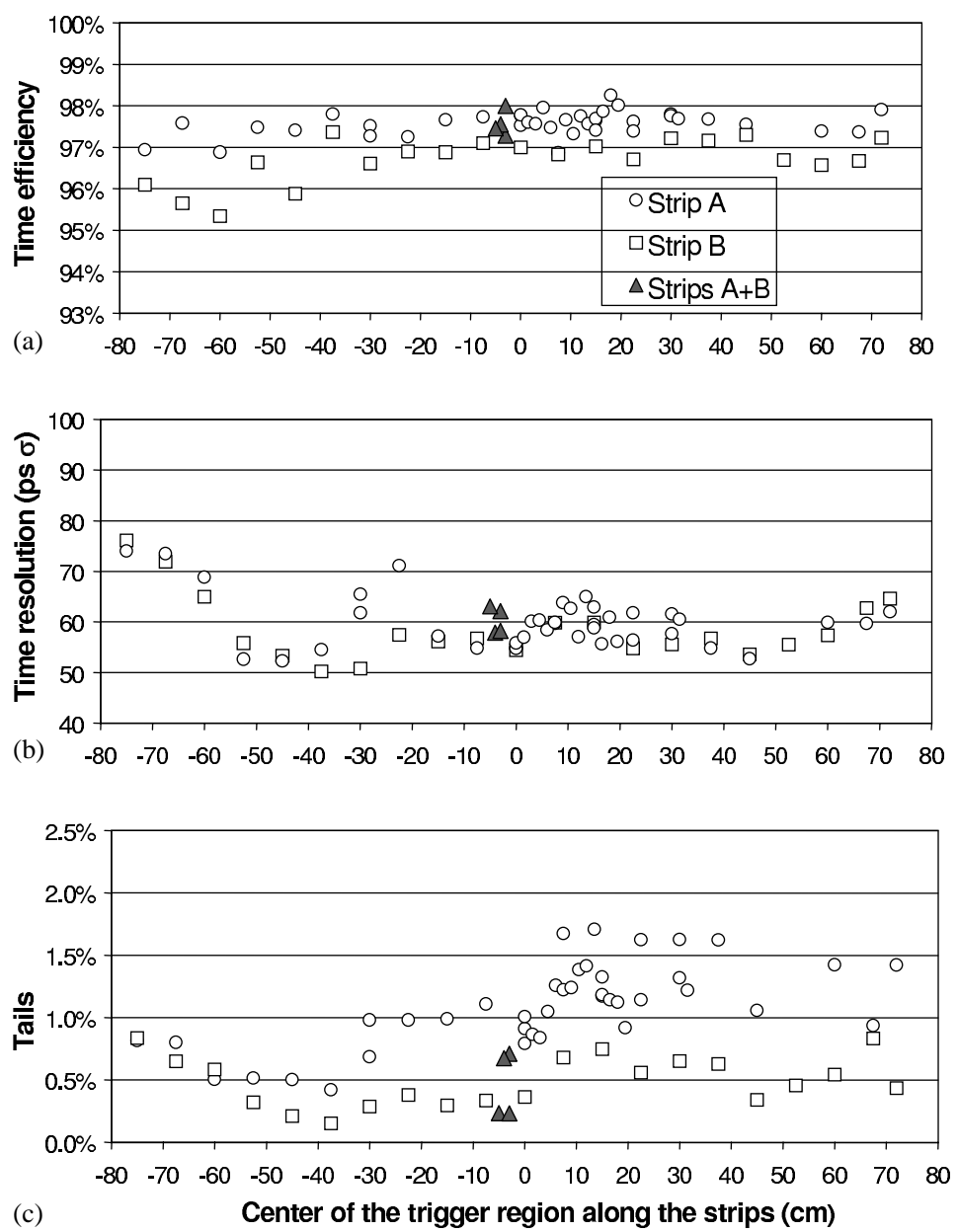

Fig. 3. Performance of the large counter as a function of the position of the centre of the trigger region along the strips: (a) efficiency for MIPs, better than $95 \%$, (b) time resolution, ranging from 50 to $75 \mathrm{ps} \sigma$; (c) timing tails, smaller than $2 \%$. In all figures the solid triangles correspond to data taken with both strips connected together, showing that there was no degradation of the performance when the active area per amplifier was doubled (to $800 \mathrm{~cm}^{2}$ per amplifier). 
signals at both ends of each strip, showing a resolution of $1.2 \mathrm{~cm} \sigma$ and a linearity error smaller than $1.4 \mathrm{~cm}[8]$.

\subsection{Position-sensitive single-gap counters}

Four single-gap counters were built and tested. Two counters were equipped with position-sensitive readout and two had the position readout electrode replaced by a single metallic plate. No difference was found between these two versions, showing that the position readout circuitry was fully compatible with the accurate measurement of time.

All counters have shown an efficiency between $70 \%$ and $80 \%$, compatible with previous measurements in single-gap counters [4]. The positionresolution was estimated to be better than $3 \mathrm{~mm}$ FWHM, which is sufficient for the present application.

The timing resolution had between 50 and $60 \mathrm{ps}$ $\sigma$, with moderate tails, with a typical distribution as shown in Fig. 4. Using the position information a data sample containing mostly events from the counter edges and corners could be selected, being observed that there is no particular performance degradation in such locations.

It is expected that the redundant information provided by a multilayer arrangement of such

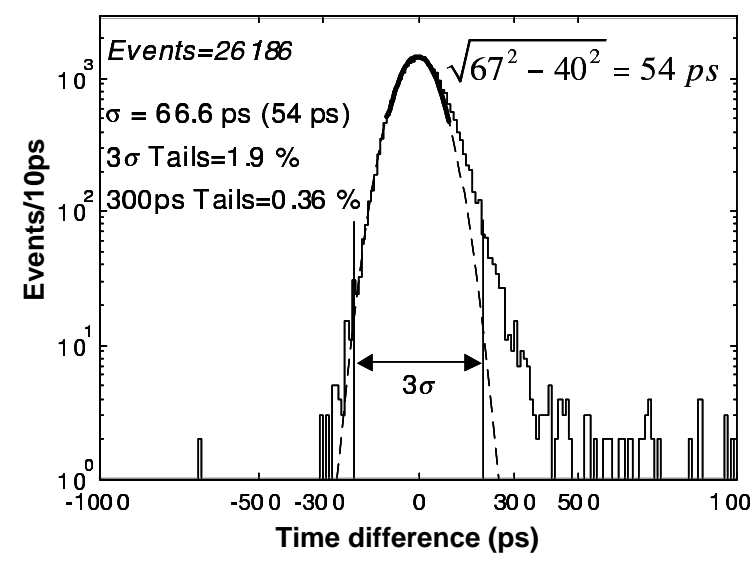

Fig. 4. Typical time distribution from a position-sensitive single-gap counter. A distribution width of $67 \mathrm{ps} \sigma$ corresponds to a time resolution of $54 \mathrm{ps} \sigma$ after quadratic subtraction of the measured $40 \mathrm{ps}$ contribution of the reference counter. Moderate timing tails are also shown.

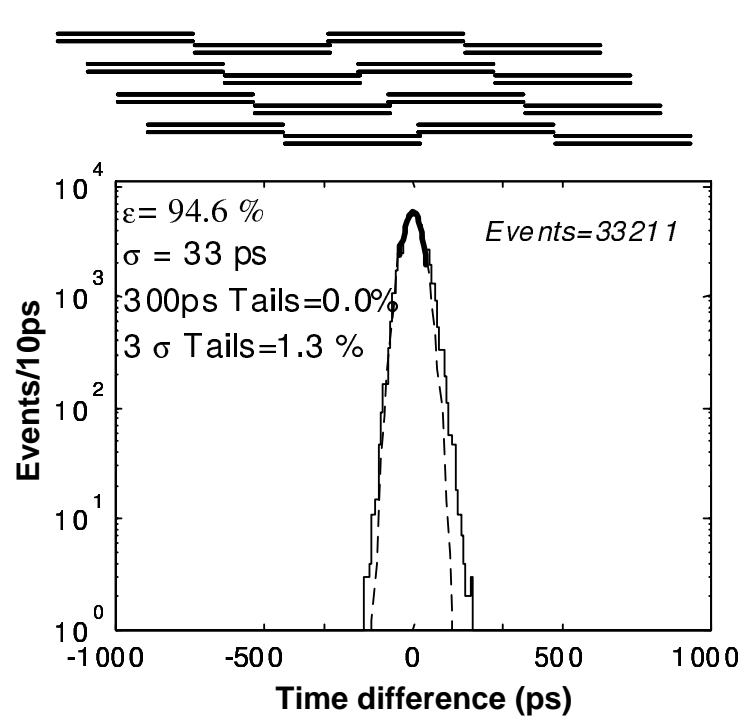

Fig. 5. Monte Carlo simulation of a 4-layer system, using for each layer the measured time response given in Fig. 4 and an efficiency of $75 \%$. The device would have an efficiency of $95 \%$ (after the rejection of tails) and a time resolution of $33 \mathrm{ps} \sigma$, essentially free of timing tails.

counters would provide a better timing resolution along with a reduction of the timing tails (after appropriate cuts). Such multilayer system can be accurately simulated from the measured single-layer data if the layers are assumed to be independent, being the results for a four layer system shown in Fig. 5. The device would have a time resolution of 33 ps $\sigma$ essentially free of timing tails and an efficiency of $95 \%$ (after the rejection of tails).

\section{Acknowledgements}

The special colaboration of Paulo Pascolati and Vito Vanin of Universidade de São Paulo, Brasil, is gratefully acknowledged.

This work was done in the framework of the FCT project CERN/P/FIS/15198/1999.

\section{References}

[1] P. Fonte, A. Smirnitski, M.C.S. Williams, Nucl. Instr. and Meth. A 443 (2000) 201.

[2] P. Fonte, R. Ferreira Marques, J. Pinhão, N. Carolino, A. Policarpo, Nucl. Instr. and Meth. A 449 (2000) 295. 
[3] A. Akindinov, et al., A four-gap glass-RPC time-of-flight array with $90 \mathrm{ps}$ time resolution, ALICE Note ALICEPUB-99-34, preprint CERN-EP-99-166.

[4] P. Fonte, V. Peskov. High-resolution TOF with RPCs, presented at the PSD99-Fifth International Conference on Position-Sensitive Detectors, University College, London, 13-17 September 1999, preprint LIP/00-04 http://arxiv. org/abs/physics/0103057, Nucl. Instr. and Meth. A 477 (2001) 17.

[5] P. Fonte, High-resolution timing of MIPs with RPCs - a model, presented at the RPC99-5th International Workshop on Resistive Plate Chambers, Bari, Italy, 28-29 October 1999, Nucl. Instr. and Meth. A 456 (2000) 6.

[6] FOPI-Collaboration, Upgrading the FOPI detector system, GSI-Scientific Report, 1998, p. 177.

[7] The HARP collaboration (PS214), The Hadron production experiment at the PS, CERN-SPSC/99-35, SPSC/ P315, 15 November 1999.
[8] A. Blanco, R. Ferreira-Marques, C. Finck, P. Fonte, A. Gobbi, A. Policarpo, M. Rozas, A large area timing RPC, Nucl. Instr. and Meth. A, to appear. Also available on: http://arxiv.ORG/ABS/PHYSICS/0103086.

[9] P. Camarri, et al., Nucl. Instr. and Meth. A 414 (1998) 317.

[10] A. Blanco, N. Carolino, P. Fonte, A. Gobbi, A simplified and accurate front-end electronics chain for timing RPCs, presented at the LEB 2000-6th Workshop on Electronics for LHC Experiments, Cracow, Poland, 11-15 September 2000, published in the Conference Proceedings CERN 2000-010 CERN/LHCC/2000-041;

A. Blanco, N. Carolino, P. Fonte, A. Gobbi, A new front-end electronics chain for timing RPCs, presented at the 2000 IEEE Nuclear Science Symposium and Medical Imaging Conference, Lyon, France, 15-20 October 2000, IEEE Trans. Nucl. Sci., accepted for publication. 